\title{
Impact of Backhauling Power Consumption on the Deployment of Heterogeneous Mobile Networks
}

\author{
Sibel Tombaz ${ }^{1}$, Paolo Monti ${ }^{2}$, Kun Wang ${ }^{3}$, Anders Västberg ${ }^{1}$, Marco Forzati ${ }^{3}$ and Jens Zander ${ }^{1}$ \\ ${ }^{1}$ Wireless@KTH, Royal Institute of Technology, KTH, Electrum 229, 16440 Kista, Sweden \\ ${ }^{2}$ NEGONET Group, Royal Institute of Technology, KTH, Electrum 229, 16440 Kista, Sweden \\ ${ }^{3}$ Acreo AB, Electrum 236, 16440 Kista, Sweden \\ Email: sibel@kth.se, pmonti@kth.se, kun.kang@acreo.se, vastberg@kth.se, \\ marco.forzati@acreo.se, jenz@kth.se
}

\begin{abstract}
Energy efficiency in cellular mobile radio networks has recently gained great interest in the research community. The development of more energy efficient hardware and software components aside, effect of different deployment strategies on energy efficiency are also studied in the literature. The latter mainly consist of optimizing the number and the location of different types of base stations in order to minimize the total power consumption. Usually, in the literature, the total network power consumption is restricted to the sum of the power consumption of all base stations. However, the choice of a specific deployment also affects the exact implementation of the backhaul network, and consequently its power consumption, which should therefore be taken into account when devising energy efficient deployment. In this paper, we propose a new power consumption model for a mobile radio network considering backhaul. We then handle a case study and perform a comparison of the power consumption of three different heterogeneous network deployments, and show how backhaul has a non-negligible impact on total power consumption, which differs for different deployments. An energy efficiency analysis is also carried out for different area throughput targets.
\end{abstract}

Index Terms - Power Consumption Model, Heterogeneous Networks, Energy Efficiency, Backhaul, Network Deployment.

\section{INTRODUCTION}

Over the last decade global warming has become an important issue on the political agenda and efforts for reducing the power consumption and, consequently, the carbon emissions is currently attracting a lot of attention [1]. In this regard the Information and Communication Technology (ICT) industry is seen as an increasingly important contributor with an $8 \%$ of the worldwide energy consumption in 2008, which is expected to double by 2020. Mobile communication networks alone consume $0.5 \%$ of the global energy consumption [2]. With the increase in the demand for broadband services that we are experiencing nowadays, there is a need for denser networks and in this context increased energy prices are expected to constitute a significant challenge in the near future [3]-[4].

Besides the environmental concerns, there is a strong economical motivation for network operators to decrease the power consumption of the network. The main consumers are data servers, backhaul routers and base stations (BS) which constitutes between 60 to $80 \%$ of the overall network power consumption [2]. Energy efficiency can be improved in two main ways. The first consists of reducing the power consumption of the main consumer, i.e., the BS (either by using more power-efficient hardware or by using more advanced software to adapt power consumption to the traffic situation). The second is intelligent network deployment strategies where using high density deployment of low power, small base stations is believed to decrease the power consumption compared to low density deployment of high power macro base stations. The idea being that a BS closer to mobile users lowers the required transmit power due to advantageous path loss conditions [5].

There are numerous papers related to energy efficiency of cellular radio networks in the literature. In [6], energyawareness of base stations is studied to decrease the power consumption when the traffic is low. The problem is handled as a network optimization problem in [3], where under-utilized part of the network is suggested to shut down based on the knowledge of the static users locations, and 50\% energy savings is claimed in less busy hours. Relaying is also proposed as a method to decrease the power consumption in [7], because of the path loss reduction property. On the other hand, the effects of different network deployment schemes are studied in [1], and [8] where the positive effect of using micro base stations on the energy efficiency is shown by simulations. In all these papers power consumption is calculated by only considering base stations while the backhaul part is ignored. However we believe that, especially for network deployment optimization, backhaul power consumption should be taken into account.

In this paper, the impact of mobile backhauling on the total power consumption of a mobile radio network is evaluated. To this end, a power consumption model for mobile radio networks including backhauling is proposed and used to compare the area power consumption of three different heterogeneous deployment schemes under the same area throughput and coverage target. We aim to answer the following questions;

- Is there a tradeoff between the power saved by using low power base stations and the excess power that has to be spent to backhaul their traffic?

- Can the effect of backhaul power consumption change the most energy efficient deployment strategy in cellular mobile networks? 


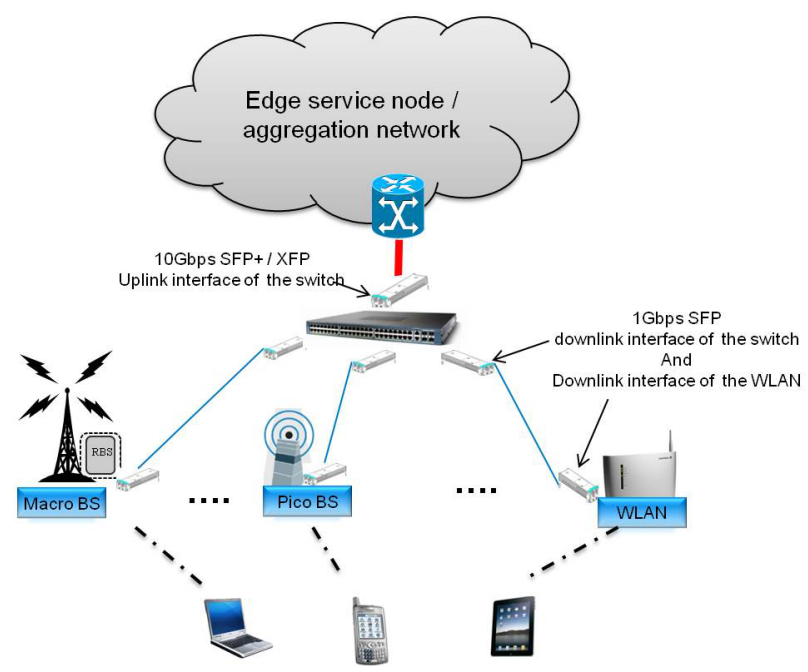

Fig. 1. Carrier Ethernet Backhauling Layout.

The paper is organized as follows. In Section II, we introduce the new power consumption model and discuss the consumers in detail. Section III presents a case study in which the power consumption of three different heterogenous networks are calculated with and without backhaul power consumption. An energy efficiency analysis is also performed. The last section concludes the paper and identifies open problems and possible future work.

\section{Wireless BAckhaul Power CONSUMPTION}

This section presents a power consumption model for cellular mobile networks including the backhauling part of the network. Different architectures and technologies are available to implement backhauling: we have chosen the solution which seems the most readily available and which presents the lowest complexity: a fiber optic network based on point-to-point Ethernet.

\section{A. Backhauling Solution}

Figure 1 shows an example of a fiber-optic carrier Ethernet backhauling solution for a heterogeneous mobile network. IP/Ethernet interfaces for backhauling has been standardized in 3GPP beyond mobile data core elements and out towards the base stations and radio controllers [9]. Ethernet switches for backhauling can be flexibly located in the distributed cell sites, or in a centralized aggregation node, or at both locations to have several levels of aggregation. In this study, for the sake of simplicity, we assumed a backhaul system with one level of aggregation only, i.e., the traffic from all wireless nodes is collected at one or more aggregation switches (if the number of the traffic streams to be aggregated so requires) just before the edge service node or the aggregation network. It is assumed that all backhaul links from all the base stations (Macro, Pico and WLAN) to the aggregation switch(es) are optical fibers. Each cell site has an optical small-form factor pluggable (SFP) interface connected to an Ethernet switch port at the aggregation site. An alternative solution would be to use copper cable connections for the downlink. However, even if this solution would be acceptable for 3G UMTS networks, copper cables would not provide enough bandwidth to support Long Term Evaluation (LTE) and LTE Beyond networks which offer $100 \mathrm{Mbps}$ to each user.

\section{B. Power Consumption Model}

Given the backhauling architecture presented in the previous section, the power consumed to backhaul the traffic from all the base stations to the aggregation network, can be expressed as follows. The average power consumption of a base station $P_{i}$ is modeled as a linear function of average radiated power, that is:

$$
P_{i}=a_{i} P_{t x}+b_{i}+c_{i},
$$

where $P_{t x}$ denotes the average radiated power. The coefficient $a_{i}$ accounts for the power consumption that scales with the transmit power due to RF amplifier and feeder losses while $b_{i}$ models the power consumed independently of the transmit power due to signal processing and site cooling. Additionally with respect to the power consumption model presented in [1], on which our model is based, a coefficient $c_{i}$ is included which represents the power consumption of the SFP used to transmit over the backhauling fiber.

Equation (1) defines the power consumed by one base station, but in order to quantify the total power consumption of a heterogeneous mobile network, the power consumed by the backhaul $\left(P_{b h}\right)$ needs to be added, as shown in the following expression:

$$
\mathcal{P}=\sum_{i=1}^{m} N_{i} P_{i}+P_{b h},
$$

where $m$ is the number of base station types used in the network, $N_{i}$ is the total number of base stations of a specific type $i$-th (e.g., Macro base stations), and $P_{i}$ is the power consumption of a base station of type $i$, which is calculated using (1).

The backhaul power $P_{b h}$ includes not only the downlink and the uplink power consumption (i.e., from a base station to the aggregation switch(es) and from the switch(es) to the aggregation network, respectively) but also the power consumed at the aggregation switch(es), which is proportional to the total traffic backhauled from the mobile network. A detailed expression for $P_{b h}$ is given by

$$
P_{b h}=\left\lceil\frac{1}{\max _{d l}}\left(\sum_{i=1}^{m} N_{i}\right)\right\rceil P_{s}+\left(\sum_{i=1}^{m} N_{i}\right) P_{d l}+N_{u l} P_{u l}
$$

where, $P_{d l}$ is the power consumed by one downlink interface in the aggregation switch used to receive the backhauled traffic. It is assumed that: 1) all switches are identical, 2) each base station in the network, regardless of its type, uses a dedicated downlink interface, and 3) all downlink interfaces are identical and use the same transmission rate. $N_{u l}$ and $P_{u l}$ are the total number of uplink interfaces, and the power 
consumption of one uplink interface, respectively. It is also assumed that all uplink interfaces are identical. $N_{u l}$ is a function of total aggregate traffic collected at the switch(es) $\left(A g_{t o t}\right)$ and the maximum transmission rate of an uplink interface $\left(U_{\max }\right)$. More formally $N_{u l}$ can be expressed as

$$
N_{u l}=\left\lceil\frac{A g_{t o t}}{U_{\max }}\right\rceil \text {. }
$$

The constant $\max _{d l}$ in (3) represents the maximum number of downlink interfaces available of the aggregation switch and it is used to compute the total number of switches that are needed to collect the backhauled traffic of the mobile network. Finally, $P_{s}$ represents the power consumed by a switch. $P_{s}$ is assumed to have two main contributors. The first one is traffic independent and models the power consumption of the backplane of the switch. The second one depends on the amount of traffic that is traversing the switch $\left(A g_{\text {switch }}\right)$. The relative influence of these power quantities is assumed to be regulated by a weighting parameter $\alpha \in[0,1]$ as shown below:

$$
P_{s}=\alpha P_{\max }+(1-\alpha) \frac{A g_{\text {switch }}}{A g_{\max }} P_{\max } .
$$

$P_{\max }$ represents the maximum power consumption of the switch, i.e., when all the downlink interfaces are in use, while $A g_{\max }$ is the maximum amount of traffic a switch can handle.

The model presented above is then used to compare the power consumption of three heterogeneous networks with and without considering the backhaul power consumption. Details on this case study are presented next.

\section{Power Consumption AnAlysis}

To asses the impact that the backhaul segment has on the network power budget, this section presents a case study where the power consumption of three, minimum-cost, heterogeneous network deployments, each one providing the same area throughput and coverage, are considered for a nonuniform traffic scenario. The underlaying system model and the assumptions used for the network deployment are made in accordance with the work presented in [10].

First this section briefly describes the network layout under consideration and the method used to obtain each deployment. Then, the area power consumption for each heterogeneous network deployment is calculated using the power consumption model introduced in Sec. II. In the presented case study, the parameter $m$ introduced in (2) is assumed to be equal to two. This means that only two types of base stations are considered for each heterogeneous network deployment, i.e., 1) Macro base stations in combination with Pico base stations, and 2) Macro base stations in combination with WLAN access points.

For benchmarking purposes, the power consumption figures calculated using the model introduced in [1] are also shown. In this benchmark model, that does not account for the power consumed by the backhauling segment, the average power consumption of a base station $P_{i}$ is modeled as follows:

$$
P_{i}=a_{i} P_{t x}+b_{i}
$$

where $a_{i}, b_{i}$ and $P_{t x}$ are defined as in Sec. II.

Additionally, performances in terms of energy efficiency are also analyzed. Energy efficiency is defined as the ratio of the total network throughput over the energy consumption within a given period, $T$, where the unit is bits/Joule [4],[11]. For an heterogeneous network using only two type of base stations, i.e., $N_{1}$ macro base station and $N_{2}$ pico base stations, the energy efficiency can be written as follows:

$$
E E=\frac{\sum_{i=1}^{N_{1}} R_{m a, i}+\sum_{i=1}^{N_{2}} R_{s, i}}{\left\{\sum_{i=1}^{N_{1}} P_{m a, i}+\sum_{i=1}^{N_{2}} P_{s, i}+P_{b h}\right\}} .
$$

$R_{m a, i}$ and $R_{s, i}$ represent the average data rate provided of a macro base station and a small, low power base station, respectively. On the other hand, $P_{m a, i}$ and $P_{s, i}$ represent the power consumed by both types of base stations. These values are obtained using (1) and $P_{b h}$ is the backhaul power consumption of the network, expressed as in (3).

\section{A. Case Study}

The doctoral thesis by Klas Johansson [12] presents a series of cost effective deployment strategies for heterogeneous wireless networks. We have used this basic method to study the power consumption of the following heterogeneous wireless networks;

- 3G UMTS based on Macro base stations,

- 3G UMTS based on Macro and Pico base stations,

- 3G UMTS based on Macro base stations and WLAN access points.

The method presented in the thesis consists of simulating spatial traffic densities per area unit with a log-normal distribution. The target QoS level has been set to an average of 1 Mbps downlink per user during busy hour for at least $\% 95$ of the users. It is assumed that each type of base station has a constant maximum supported throughput $s_{\max }\left[\mathrm{Mbps} / \mathrm{km}^{2}\right]$ and a maximum range $\delta_{\max }[\mathrm{km}]$. The number of required base stations for a target set of values of both coverage and throughput is determined sequentially, taking the values for the non-uniform traffic as an input. The details of the simulation can be found in the thesis. It should be noted that, in [12] the network deployment is optimized by taking cost-effectiveness as a objective function only, where the energy cost is only marginally considered.

TABLE I

Characteristics of Base Stations[12]

\begin{tabular}{cccc}
\hline \hline Parameter & Macro BS & Pico BS & WLAN \\
\hline Sectors per base station & 3 & 1 & 1 \\
Max range $\delta_{\max }[\mathrm{m}]$ & $2000 / \sqrt{3}$ & $200 / \sqrt{3}$ & $100 / \sqrt{3}$ \\
Transmitter power $P_{t x}[\mathrm{dBm}]$ & 43 & 30 & 23 \\
\hline
\end{tabular}

The characteristics of the base stations considered in the study are summarized in Table I. Macro base stations are assumed to have 3-sectors with antennas above roof-top for longer range. They have a maximum of three carriers per sector. On the other hand, Pico base stations and WLAN access points have single carrier and omni-directional antennas. 


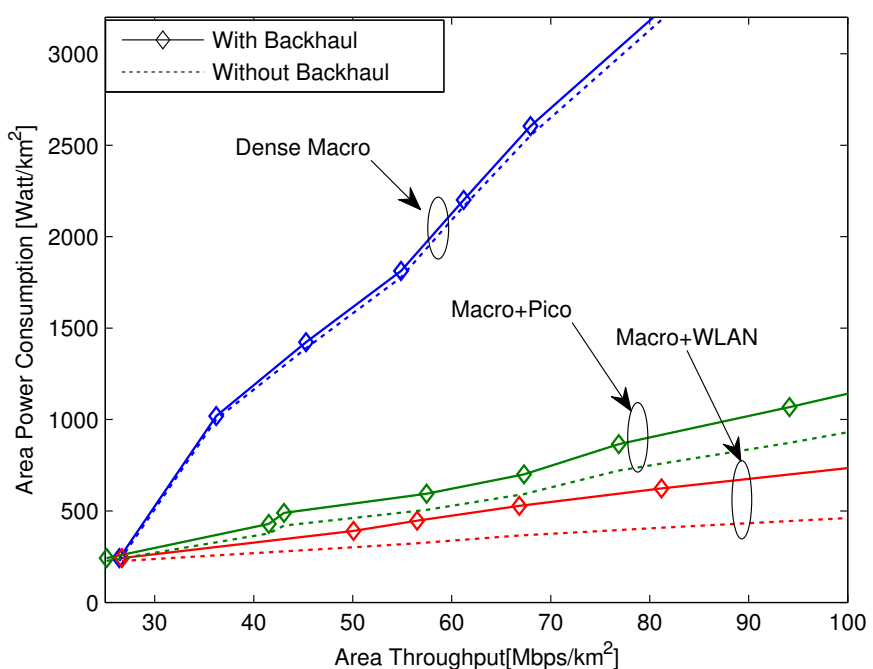

Fig. 2. Area power consumption as a function of target area throughput.

\section{B. Power consumption comparison}

In this section, the total power consumption of the three heterogeneous deployments scenarios presented previously are compared. All three deployments have the same characteristics in terms of coverage and capacity, and they just differ in the number and in the type of base stations used. In order to compute the power consumption of the backhauling segment the values in Table II are used, while the power consumption parameters of each type of base station are summarized in Table III. The transmission speed for the transmitters and the receivers at the downlink interface is assumed to be $1 \mathrm{~Gb} / \mathrm{s}$, while the maximum transmission speed for the uplink interface $\left(U_{\max }\right)$ is $10 \mathrm{~Gb} / \mathrm{s}$. The parameter $\alpha$ and $\max _{d l}$ are equal to 0.9 and 24 respectively, while $A g_{\max }$ is equal to $24 \mathrm{~Gb} / \mathrm{s}$.

TABLE II

POWER CONSUMPTION PARAMETERS FOR BACKHAUL

\begin{tabular}{cc}
\hline \hline Consumers & Power Consumption[W] \\
\hline$P_{\max }$ & 300 \\
$P_{u l}$ & 2 \\
$P_{d l}$ & 1 \\
$c_{i}$ & 1 \\
\hline
\end{tabular}

TABLE III

Power Consumption Parameters for Base Station[1],[11]

\begin{tabular}{ccc}
\hline \hline$i$ (Base Station Type) & $a_{i}$ & $b_{i}$ \\
\hline Macro & 21.45 & 354.44 \\
Pico & 5.5 & 38 \\
WLAN & 3.2 & 10.2 \\
\hline
\end{tabular}

In Fig.2 the impact of backhaul on the overall power consumption of the network is shown for three different network deployment schemes. The power is computed in terms of area power consumption and is presented as a function of the area throughput. As it can be seen, the relative effect of the backhaul power consumption is increasing as the more

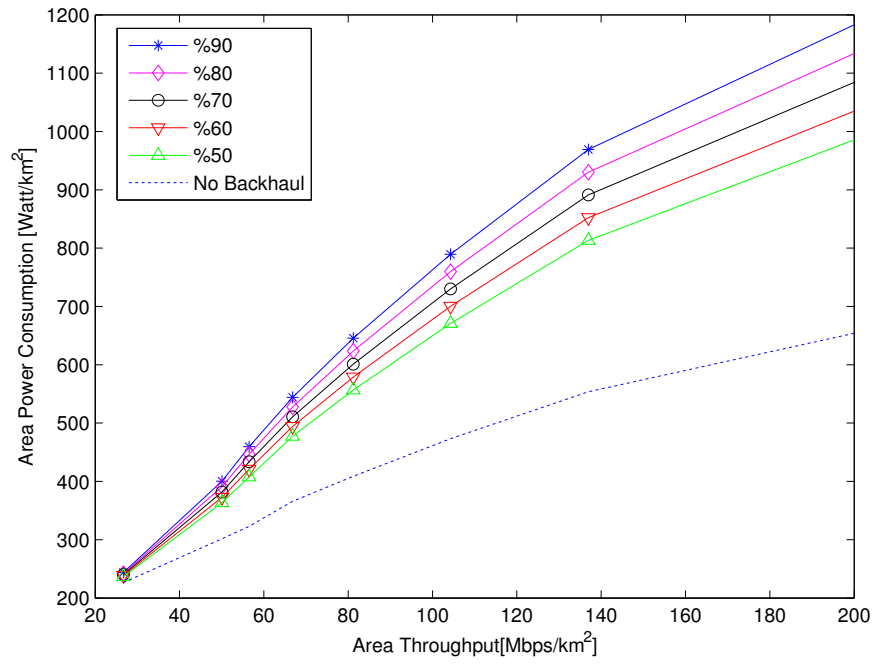

Fig. 3. Sensitivity of area power consumption based on switch power's traffic dependency for Macro+WLAN deployment scenario.

small, low power base stations are used. Especially for the Macro+WLAN deployment scenario, the total network power consumption is almost doubled and with a rate that increases with higher area throughput values. However the effect of backhaul is not as significant when it comes to the case of Dense Macro deployment where too many additional base stations are not needed to satisfy the required area throughput, as it was for the Macro+WLAN case. This result indicates the trade-off between the power saved using low power base stations and the excess power that spent for backhaul. On the other hand, even with the inclusion of the extra power consumed by the backhaul segment, the use of low power WLAN access points and pico base station still remains the best choice to have a network with an overall reduced power consumption. It should be noted that the presented network deployment results are not optimized in terms of power consumption as explained in the previous section. The moment power consumption (including backhaul) is considered as part of energy based optimization process, it would be expected that overall power figure for all three deployments will be lower as well as the gap between each deployment strategy.

With the development of new power saving techniques in IP/Ethernet switches the traffic dependent portion of power consumption would gradually play a more important roles in the future. In Fig. 3 we assumed five different $\alpha$ values which reflects the percentage of the switch power that is independent of network traffic for Macro+WLAN deployment scenario. As we can see, with an increasing energy efficiency of the switchs (decreasing of $\alpha$ values), the impact of backhaul on the overall network power consumption decreases.

Table IV compares the energy efficiency of different network deployments for two different target area throughput values. As it can be expected, macro base stations combined with WLAN provide the highest energy efficiency for both area throughput targets, compared to the Dense Macro and 
the Macro+Pico case. Another important conclusion is the difference between energy efficiency results for different target area throughputs, for the same deployment case. As it is shown in Table IV, energy efficiency is decreasing with an increased area throughput for the Dense Macro case, while it increases when Macro+WLAN are used. These results can be explained as follows; with the Dense Macro case, the increased capacity can not compensate the additional power consumption of macro base stations. However, lower power consumption advantage of WLAN reverse this conclusion for macro+WLAN deployment scenario.

TABLE IV

ENERGY EFFICIENCY (MBITS/WATT) COMPARISON FOR DIFFERENT AREA THROUGHPUT

\begin{tabular}{ccc}
\hline \hline Layout & $\approx 40 \mathrm{Mbps} / \mathrm{km}^{2}$ & $\approx 80 \mathrm{Mbps} / \mathrm{km}^{2}$ \\
\hline Dense Macro & 0.0318 & 0.0249 \\
Macro+Pico & 0.0966 & 0.0889 \\
Macro+WLAN & 0.1280 & 0.1302 \\
\hline
\end{tabular}

\section{CONClusion And Future Work}

In this paper, we proposed a new power consumption model for mobile radio network where the backhaul power is taken into account. The impact of backhaul on the total power consumption was shown, by means of numerical simulations, to be significant for three heterogeneous network deployments (i.e using only Macro base stations, Macro and Pico base stations, Macro base stations and WLAN access points).

For the cases where small low power base stations are used to increase the capacity of the network, the relative effect of backhaul power consumption is getting more influential. This indicates a tradeoff between the power saved by using low power base stations and the excess power that has to be spent to backhaul their traffic. However, it is shown that this impact is not enough to shift the energy efficient deployment toward larger cells. We may expect larger backhaul impact on the energy-optimized networks and cost-optimized networks which consider significant increase of energy cost. Moreover, a sensitivity analysis of the proposed power consumption model is performed based on the switch power's traffic dependency. We also investigated different energy efficiency behavior for heterogeneous networks under different area throughput targets which is directly related to their required transmitter power-capacity enhancement relations. The results presented here are obtained for a specific backhaul solution, and may differ for alternative solutions, but the underlining message is that when assessing the benefits of a deployment strategies, the backhaul power consumption can not be simply ignored.

As future work, we plan to improve the power consumption model of ethernet switches by considering the scalability (i.e., with the possibility to switch off individual ports rather than whole board which may be expected from future switches) and use different backhaul network architectures with less general assumptions (e.g., using nonidentical switches, decentralized aggregation node).

\section{ACKNOWLEDGMENT}

The authors would like to offer our gratitude to Klas Johansson for sharing his simulation results. We would like to thank Mats Nilsson, Lena Wosinska and Andreas Aurelius for their help and support in making this work possible. The work presented in this paper was carried out with the support of the Energy-efficient Wireless Networking (eWIN) and Optical Networking System (ONS) focus projects, part of ICT - The Next Generation (TNG) Strategic Research Area (SRA) initiative at the Royal Institute of Technology.

\section{REFERENCES}

[1] F. Richter, A. Fehske, and G. Fettweis, "Energy efficiency aspects of base station deployment strategies for cellular networks," in Proc. of IEEE Vehic. Technol. Conf. (VTC Fall), Anchorage,USA, September 2009, pp. $1-5$.

[2] M. Pickavet, W. Vereecken, S. Demeyer, P. Audenaert, B. Vermeulen, C. Develder, D. Colle, B. Dhoedt, and P. Demeester, "Worldwide energy needs for ict: The rise of power-aware networking," in Proc. of International Symposium on Advanced Networks and Telecommunication Systems (ANTS'08), Bombay, India, December 2008, pp. 1-3.

[3] K. Dufková, M. Bjelica, B. Moon, L. Kencl, and J. L. Boudec, "Energy savings for cellular network with evaluation of impact on data traffic performance," in Proc. of European Wireless Conference, Lucca, Italy, April 2010, pp. 916-923.

[4] Y. Chen, S. Zhang, S. Xu, and G. Y. Li, "Fundamental tradeoffs on green wireless networks," Accepted to IEEE Communications Magazine, 2011.

[5] A. Fehske, F. Richter, and G. Fettweis, "Energy efficiency improvements through micro sites in cellular mobile radio networks," in Proc. of IEEE Global Telecommun. Conf. (GLOBECOM), Hawaii, USA, December 2009, pp. 1-5.

[6] M. Marsan, L. Chiaraviglio, D. Ciullo, and M. Meo, "Optimal energy savings in cellular access networks," in Proc. of IEEE Int. Conf. Commun. (ICC), Dresden, Germany, June 2009, pp. 1-5.

[7] P. Herhold, W. Rave, and G. Fettweis, "Relaying in cdma networks: Pathloss reduction and transmit power savings," in Proc. of IEEE Vehic. Technol. Conf. (VTC Spring), Jeju, Korea, April 2003, pp. 2047 - 2051.

[8] F. Richter, A. J. Fehske, P. Marsch, and G. P. Fettweis, "Traffic demand and energy efficiency in heterogeneous cellular mobile radio networks," in Proc. of IEEE Vehic. Technol. Conf. (VTC Spring), Taipei, Taiwan, May 2010, pp. 1-6.

[9] T. System Aspects, "Sae architecture revisit and technical review," 3rd Generation Partnership Project, Tech. Rep., 2007.

[10] K. Johansson, J. Zander, and A. Furuskär, "Modelling the cost of heterogeneous wireless access networks," Int. J. Mobile Network Design and Innovation, 2007.

[11] W. Wang and G. Shen, "Energy efficiency of heterogeneous cellular network," in Proc. of IEEE Vehic. Technol. Conf. (VTC Fall), Ottawa, Canada, Sept 2010, pp. 1-5.

[12] K. Johansson, "Cost effective deployment strategies for heterogeneous wireless networks," Ph.D. dissertation, pp. Stockholm, Sweden, November, 2007, kTH Information and CommunicationTechnology. 